\title{
Sequential Therapies and the Cost-Effectiveness of Treating Metastatic Colon Cancer Patients
}

\author{
Andinet Woldemichael, PhD; Eberechukwu Onukwugha, MSc, PhD; \\ Brian Seal, RPh, MBA, PhD; Nader Hanna, MD; and C. Daniel Mullins, PhD
}

\begin{abstract}
BACKGROUND: Technological advances in colon cancer treatment have significantly increased survival outcomes among metastatic patients. With different chemotherapy and biologic regimens administered in first, second, and subsequent lines of treatments, costs and survival outcomes vary considerably. However, there is little evidence on how the type of regime administered in the first line of treatment affects the costs and survival outcomes of the second line of treatment.
\end{abstract}

OBJECTIVE: To examine how the cost-effectiveness of second-line treatment for elderly metastatic colon cancer patients varies by the type of regimen administered in the first line of treatment.

METHODS: The Surveillance, Epidemiology and End Results (SEER) cancer registry was used, which is linked with the Medicare claims database, to study elderly metastatic patients diagnosed between 2003 and 2009. Average survivals are estimated using the robust nonparametric KaplanMeier method. Selection bias was adjusted for using inverse probability weighting and censoring using robust nonparametric methods of estimating the average of total health care costs.

RESULTS: Mean incremental survival was 6.7 months for patients who received second-line treatment $(95 \% \mathrm{Cl}=5.7-7.7)$ compared with those receiving only first-line treatment. However, the mean incremental survival varied between 4 months $(95 \% \mathrm{Cl}=0.0-7.3)$ and 9 months $(95 \% \mathrm{Cl}=6.5-11.0)$ depending on whether fluorouracil with or without leucovorin, irinotecan, oxaliplatin, or other agents were administered in first-line treatment. The mean incremental cost associated with receipt of second-line treatment was $\$ 60,231(95 \% \mathrm{Cl}=52,461-64,198)$ but ranged between $\$ 55,368(95 \%$ $\mathrm{Cl}=48,294-61,290)$ and $\$ 71,211(95 \% \mathrm{Cl}=43,168-99,667)$, depending on the type of regimen administered in the first-line treatment. Combining survival benefits and costs, the incremental cost-effectiveness ratios per life-year gained associated with the receipt of second-line treatment were $\$ 97,368(95 \% \mathrm{Cl}=80,415-117,965) ; \$ 110,621(95 \% \mathrm{Cl}=89,560-133,961)$; $\$ 130,689(95 \% \mathrm{Cl}=101,459-171,918) ;$ and $\$ 247,951(95 \% \mathrm{Cl}=112,629-$ 808,976 ) when irinotican, fluorouracil/leucovorin, oxaliplatin, and "other" combinations were, respectively, administered in first-line treatment. In addition, the results varied depending on which statistical method was used.

CONCLUSIONS: When therapies are administered in a sequential manner, the cost-effectiveness of the second line of therapy depends on what was administered in the first line of therapy.

J Manag Care Spec Pharm. 2016;22(6):628-39

Copyright $\odot 2016$, Academy of Managed Care Pharmacy. All rights reserved.

\section{What is already known about this subject}

Studies show that when metastatic colon cancer patients are treated with a combination of chemotherapies, survival increases to 21 months; biologics alone or in combination with chemotherapy may further improve progression-free survival.
According to the National Comprehensive Cancer Network (NCCN) Practice Guidelines in Oncology, therapies are administered sequentially with important implications for survival and cost outcomes.

\section{What this study adds}

This study examined how the cost-effectiveness of second-line treatment for elderly metastatic colon cancer patients varied by the type of regimen administered in the first line of treatment. Population-level data were used from the Surveillance, Epidemiology, and End Results cancer registry linked with Medicare claims (SEER-Medicare) to identify the sequence of therapies administered to metastatic colon cancer patients.

The results highlighted that cost-effectiveness of the second line of therapy depends on what was administered in the first line of therapy.

A dvances in chemotherapies and targeted drugs have significantly increased survival outcomes among metastatic colon cancer patients. Studies show that, without chemotherapy, metastatic patients survive for only a median duration of 8 months. ${ }^{1}$ When treated with a combination of chemotherapies such as fluorouracil (5-FU), irinotecan (IRI), and oxaliplatin (OX), survival increases to 21 months. ${ }^{1}$ In addition, there is evidence that biologics in combination with chemotherapy further improves survival outcome. For instance, 1 study found that progression-free survival rates in a group who received bevacizumab (BEV) plus IRI and 5-FU/ LV (IFL) was 10.6 months compared with 6.2 months in the group administered with IFL plus placebo. ${ }^{2}$ While BEV and ziv-aflibercept (Zaltrap) block the growth of blood vessels to tumors, cetuximab (CET) and panitumumab (PAN) are epidermal growth factor receptor (EGFR) inhibitors, which block effects of hormone-like factors that promote cancer cell growth.

According to the National Comprehensive Cancer Network (NCCN) Practice Guidelines in Oncology, chemotherapies alone, or in conjunction with biologics, are sequentially administered in first line, second line, and subsequent lines of treatments depending on a patient's tolerance of toxicity, gene mutation profile, tumor progression, and prognosis. Sequential treatment protocol has important implications for survival and cost outcomes. Studies show that the type of regimen administered in the 
TABLE 1 Summary Statistics of First- and Second-Line Regimens with Socioeconomic and Clinical Characteristics $^{\mathrm{a}}$

\begin{tabular}{|c|c|c|c|}
\hline & \multirow[b]{2}{*}{$\begin{array}{c}\text { Whole Sample } \\
\quad(n=4,513)\end{array}$} & \multicolumn{2}{|c|}{ Second-Line Treatment } \\
\hline & & $\begin{array}{l}\text { Not Received } \\
(\mathrm{n}=2,566)\end{array}$ & $\begin{array}{c}\text { Received } \\
(\mathrm{n}=1,947)\end{array}$ \\
\hline \multicolumn{4}{|l|}{ First-line regimen } \\
\hline 5-FU/LV & 31 & 35 & 25 \\
\hline IRI & 14 & 15 & 14 \\
\hline OX & 51 & 46 & 59 \\
\hline Other (IROX, biologics) & 4 & 5 & 2 \\
\hline \multicolumn{4}{|l|}{ Second-line regimen } \\
\hline IRI & NA & NA & 57 \\
\hline $\mathrm{OX}$ & NA & NA & 22 \\
\hline Other (5FU/LV, IROX, biologics) & NA & NA & 21 \\
\hline \multicolumn{4}{|l|}{ Socioeconomic characteristics } \\
\hline Age at diagnosis (years), mean (SD) & $75.2(6.2)$ & $76.0(6.3)$ & $74.1(5.7)$ \\
\hline Married & 57 & 53 & 62 \\
\hline Male & 49 & 48 & 51 \\
\hline White & 81 & 80 & 82 \\
\hline Black & 10 & 10 & 8 \\
\hline Hispanic & 5 & 5 & 5 \\
\hline Other race/ethnicity & 5 & 5 & 5 \\
\hline Urban residence & 89 & 89 & 90 \\
\hline Received state assistance (buy-ins) following diagnosis & 11 & 10 & 12 \\
\hline \multicolumn{4}{|l|}{ Clinical characteristics } \\
\hline \multicolumn{4}{|l|}{ Charlson Comorbidity Index scores } \\
\hline 0 & 58 & 54 & 62 \\
\hline 1 & 23 & 24 & 22 \\
\hline $2+$ & 19 & 22 & 16 \\
\hline Not available & 5 & 6 & 4 \\
\hline \multicolumn{4}{|l|}{ Tumor size } \\
\hline Not available & 25 & 28 & 22 \\
\hline $0-39 \mathrm{~mm}$ & 20 & 19 & 22 \\
\hline $40-59 \mathrm{~mm}$ & 29 & 28 & 31 \\
\hline $60-99 \mathrm{~mm}$ & 22 & 21 & 22 \\
\hline $100+\mathrm{mm}$ & 3 & 4 & 3 \\
\hline Poorly differentiated tumor & 29 & 30 & 27 \\
\hline Poor baseline performance & 13 & 15 & 12 \\
\hline
\end{tabular}

first-line treatment significantly affects the likelihood of receiving a second-line treatment. ${ }^{3}$ Similarly, survival and cost outcomes of second-line treatment could depend on the type of regimen administered in the first-line treatment.

There is little evidence, however, on how the type of firstline regimen affects survival and cost outcomes of second-line treatment. The literature focuses on the cost-effectiveness of second-line treatment without regard to the type of regimen administered as the first line of treatment. ${ }^{4-7}$ The existing studies compare survival and cost outcomes associated with different regimens in the second-line treatment only. These outcomes are also based on randomized controlled trials (RCTs) conducted in non-U.S. health care systems and study populations. Furthermore, since elderly individuals are often underrepresented in clinical studies, it can be problematic to generalize the results from RCTs for this high risk-population within the United States.

The purpose of this study was to investigate how the costeffectiveness of second-line treatment for metastatic colon cancer patients in the U.S. Medicare population varies by the type of first-line regimens.

\section{Methods}

\section{Data and Descriptive Analysis}

For this investigation, population-level data were used from the Surveillance, Epidemiology, and End Results (SEER) 


\section{A. Overall: Anchoring Date Is Diagnosis Date}

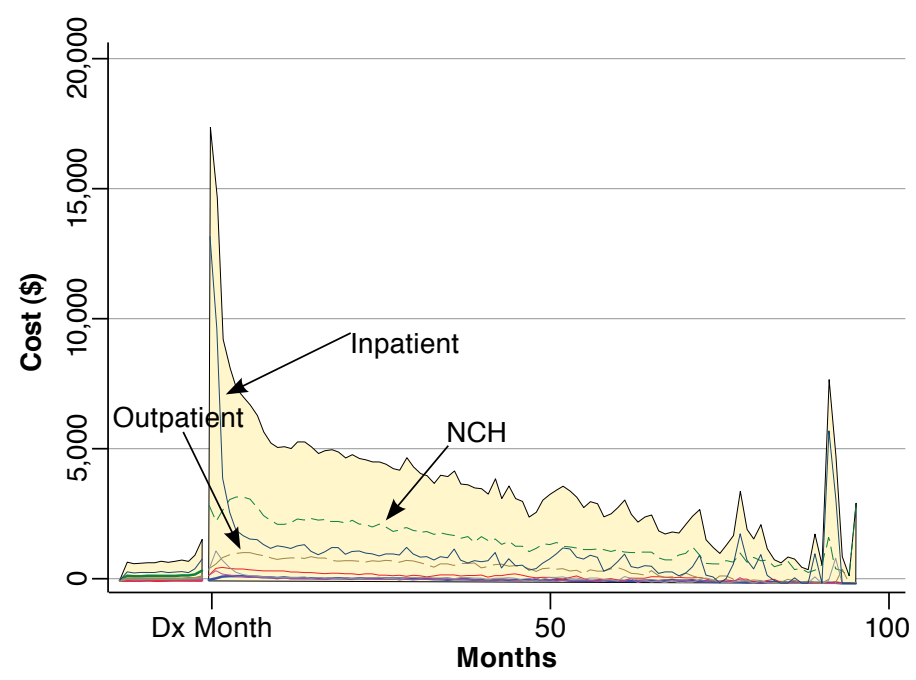

B.Second-Line Treatment Status: Anchoring Date Is Last Date of Receiving First-Line Tx Plus 1 Day

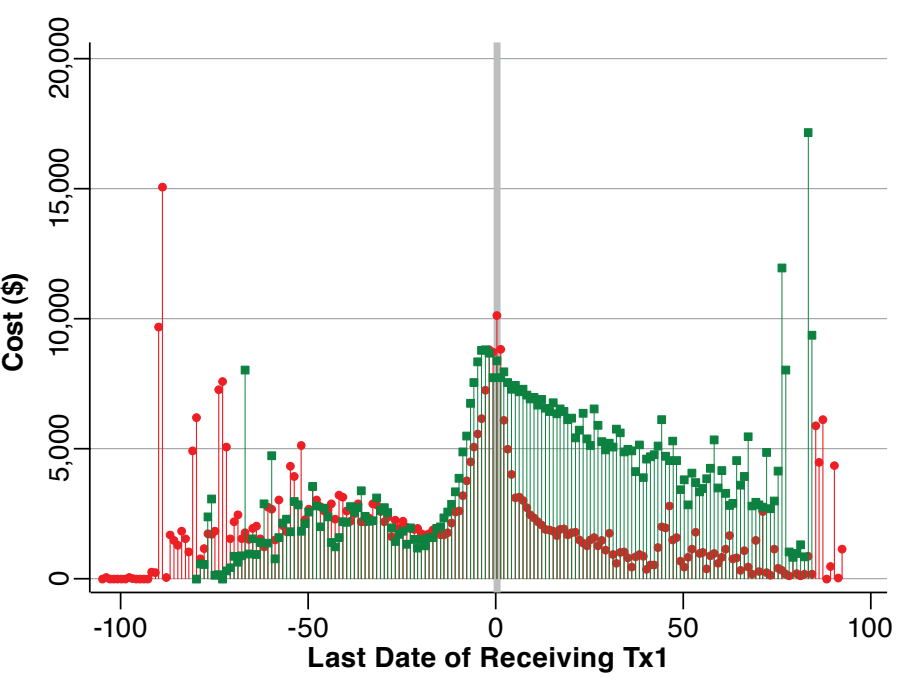

Note: All costs are in January 2009 prices. Green (squared) droplines denote monthly average total costs for patients who received second-line treatment. Red (circle) droplines denote monthly total costs for patients who did not receive second-line treatment.

$D x=$ diagnosis; $\mathrm{NCH}=$ National Claims History records; $\mathrm{T} x \mathrm{l}=$ first-line treatment; $\mathrm{T} x=$ treatment .

cancer registries linked with the Medicare claims database from the National Cancer Institute (NCI). The SEER database collects clinical, demographic, and cause of death information; the Medicare data provide claims information from hospital, physician, outpatient, home health care, hospice, and Medicare Part D. ${ }^{8}$ The linked database has clinical, demographic, socioeconomic, and claims information for 1.6 million Medicare beneficiaries diagnosed with various types of cancers. A cohort of 11,010 elderly (aged 66 and above) patients were identified who were diagnosed with metastatic colon cancer between 2003 and 2009. Of the identified cohort members, 4,513 received NCCN-recommended regimens for first-line treatment. While $43.1 \%(n=1,947)$ of cohort members received second-line treatment, $56.9 \%(n=2,566)$ of cohort members did not.

Different lines of treatments and regimens were identified using a claims-based algorithm. ${ }^{9}$ The algorithm used Current Procedural Terminology (CPT), Health Care Common Procedural Codes (HCPCs), International Classification of Diseases, Ninth Revision, Clinical Modification (ICD-9-CM) codes, and International Classification of Diseases, Ninth Revision (ICD-9) codes along with NCCN-recommended treatment cycles to identify therapies and lines of treatments from claims data. The sample was stratified into 4 groups based on the type of regimens received in first-line treatment: (1) 5-FU with or without leucovorin (5-FU/LV); (2) IRI with or without 5-FU/LV; (3)
OX with or without 5-FU/LV; and (4) "others," which included combinations such as irinotecan plus oxaliplatin (IROX) or biologics without OX, IRI, or 5-FU/LV. Table 1 presents the percentage of patients who received different types of regimens and descriptive statistics of selected patient-level clinical, demographic, and socioeconomic characteristics for the whole sample and the subsamples stratified by second-line treatment status. OX is the most frequently prescribed first-line regimen, which was administered to more than half of the patients (51\%) in the sample, followed by 5-FU/LV (31\%) and IRI (14\%). Other combinations were administered to only $4 \%$ of patients for first-line treatment.

In terms of patient-level characteristics, those who proceeded to second-line treatment were 2 years younger at the time of diagnosis than patients who did not receive second-line treatment. The descriptive statistics show that whites and married patients were more likely to receive second-line treatment. Patients who resided in urban areas and those who received financial assistance in the form of state buy-ins after diagnosis were also more likely to receive second-line treatment. Patients with 1 or more comorbidity, as measured by the Charlson Comorbidity Index (CCI) were less likely to receive secondline treatment than patients with no comorbidities. Similarly, patients who received second-line treatment had better baseline performance status and larger and well-differentiated primary tumors. 


\section{A. Propensity Scores}

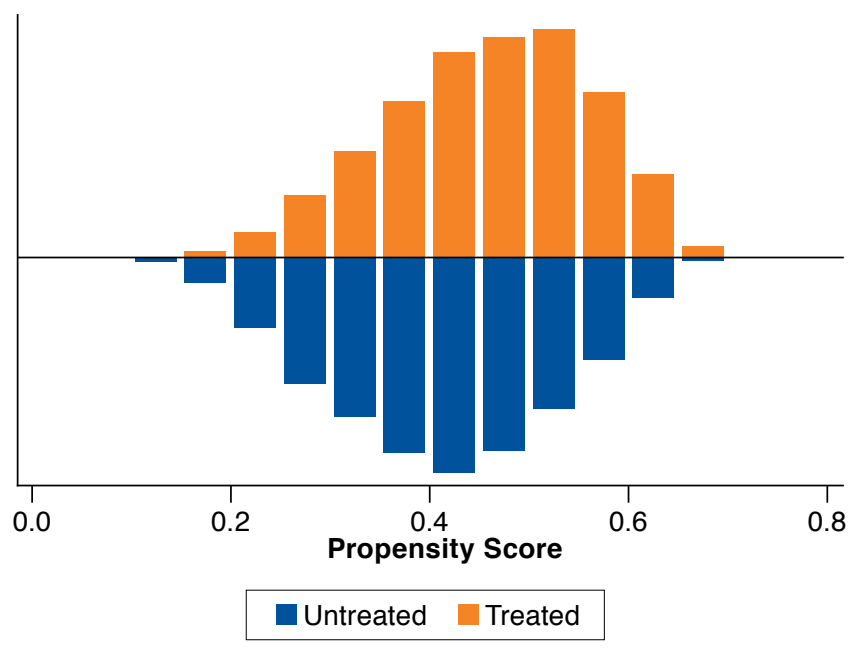

B. Normalization of IPTW

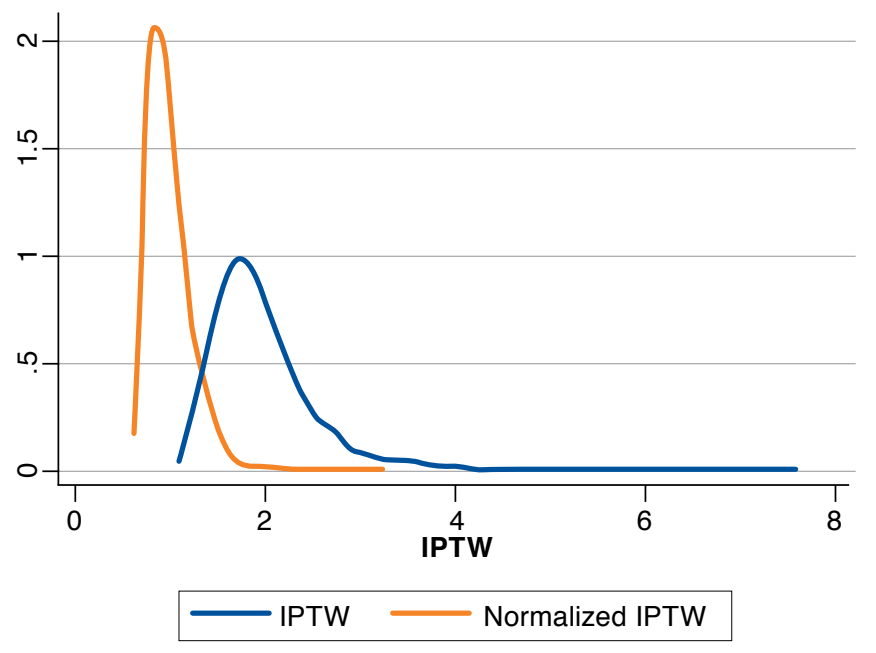

IPTW = inverse-probability-of-treatment weighting.

\section{Estimation Methods}

To estimate average total costs, selection bias into and out of receipt of second-line treatment was controlled for using the inverse-probability-of-treatment weighting (IPTW) approach. Censoring was addressed using nonparametric methods proposed by Bang and Tsiatis (2000), referred to here as the B\&T method, and Lin et al. (1997), referred to here as Lin's method. ${ }^{10,11}$ Although there are various parametric methods of adjusting survival for covariates in the case of nonproportional hazard rates, unadjusted average survival was estimated using the nonparametric Kaplan-Meier (KM) method.

The study period was restricted to a 5 -year follow-up period after the patient's last date of receiving first-line treatment plus 1 day. The event of interest is death from any cause. Patients who were alive at the end of the follow-up period, who were enrolled in health management organizations (HMOs), or who had lost Medicare Part A or Part B coverage were censored. These observations accounted for $17.7 \%(n=345)$ of patients who received second-line treatment. Similarly, 20.3\% $(n=531)$ of patients who did not receive second-line treatment were censored. The overwhelming majority of patients who were censored were censored because they were alive at the end of the study. The full cost and survival information for the censored individuals not in the dataset were not observed, which could potentially bias the estimation of average total cost, survival, and incremental costs and effects.

Estimation of Costs and Survivals. Costs were measured over a 5-year follow-up period from the anchoring date, which was set at the last date of receiving first-line treatment plus 1 day. Costs were obtained from Medicare reimbursement for hospital care, physician and clinical services, skilled nursing facility (SNF), home health agency (HHA), and hospice care. Total costs were calculated by summing reimbursements for Medicare Part A and Part B, which represented payments made to inpatient, SNF, HHA, outpatient, hospice, durable medical equipment (DME), and carrier claims, also known as Medicare National Claims History ( $\mathrm{NCH}$ ) records. Payments made for prescription drugs (Part D) were excluded from cost calculations because Part D coverage started in July 2006, whereas this study covers the period between 2003 and 2009. However, excluding Part D from the total costs for metastatic colon cancer patients in both groups had no major impact on the cost-effectiveness analysis, since it represents a small proportion of total cost (Figure 1A). The bulk of total cost comes from Medicare Part A and B reimbursements.

To make costs comparable over time, all costs were inflated to January 2009 prices using the national U.S. Bureau of Labor Statistics monthly itemized medical care component of the Consumer Price Index. Nursing home services prices were used to adjust hospice, SNF, and HHA claims; inpatient hospital prices were used to adjust inpatient claims; outpatient hospital prices were used to adjust outpatient claims; medical care commodities prices were used to adjust DME claims; and physician services prices were used to adjust $\mathrm{NCH}$ claims. Average total monthly cost was mainly driven by inpatient, $\mathrm{NCH}$ (physician and suppliers), and outpatient claims (Figure 1A). The average total monthly cost for patients who received 
TABLE 2 Estimated Survivals and Costs Associated with Receipt of Second-Line Treatment

\begin{tabular}{|c|c|c|c|c|c|c|}
\hline & & \multirow[b]{2}{*}{ Method } & \multicolumn{2}{|c|}{ Baseline } & \multicolumn{2}{|c|}{ IPTW } \\
\hline & & & Mean & $95 \% \mathrm{CI}$ & Mean & $95 \% \mathrm{CI}$ \\
\hline \multirow{3}{*}{ Survival (months) } & Received second-line Tx & Kaplan-Meier & 16.9 & $16.3-17.6$ & & \\
\hline & No second-line $\mathrm{Tx}$ & Kaplan-Meier & 10.2 & $9.5-11.0$ & & \\
\hline & Incremental survival & Kaplan-Meier & 6.7 & $5.7-7.7$ & & \\
\hline \multirow{9}{*}{ Cost, \$ } & \multirow{3}{*}{ Received second-line Tx } & Simple average & 103,711 & 94,923-107,512 & 89,322 & $81,697-92,476$ \\
\hline & & $B \& \mathrm{~T}^{\mathrm{a}}$ & 117,686 & $108,998-122,109$ & 100,530 & $93,064-104,088$ \\
\hline & & $\operatorname{Lin}^{\mathrm{b}}$ & 114,076 & $105,212-118,487$ & 97,470 & $89,957-101,020$ \\
\hline & \multirow{3}{*}{ No second-line $\mathrm{Tx}$} & Simple average & 35,068 & $34,003-36,192$ & 35,877 & $34,755-37,160$ \\
\hline & & $\mathrm{B} \& \mathrm{~T}^{\mathrm{a}}$ & 41,308 & $39,936-42,748$ & 41,963 & $40,561-43,501$ \\
\hline & & $\operatorname{Lin}^{\mathrm{b}}$ & 36,588 & $35,260-37,920$ & 37,240 & $35,859-38,721$ \\
\hline & \multirow{3}{*}{ Incremental cost } & Simple average & 68,642 & $59,764-72,745$ & 53,445 & $45,946-56,883$ \\
\hline & & $\mathrm{B} \& \mathrm{~T}^{\mathrm{a}}$ & 76,377 & $67,319-81,288$ & 58,567 & $50,877-62,558$ \\
\hline & & $\operatorname{Lin}^{\mathrm{b}}$ & 77,488 & $68,289-82,351$ & 60,231 & $52,461-64,198$ \\
\hline \multicolumn{7}{|c|}{ 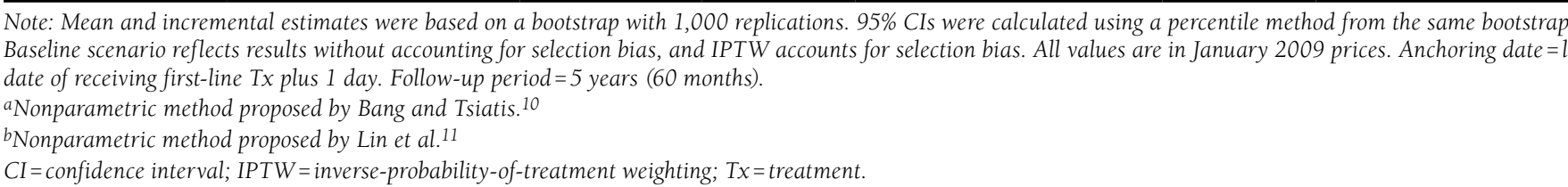 } \\
\hline
\end{tabular}

second-line treatment was considerably higher than patients who did not receive second-line treatment (Figure 1B).

The estimated average costs for censoring were adjusted using partitioned B\&T and Lin's methods. One-month interval partition over a follow-up period of 60 months was used. This sentence comes from Results section page 7 line 5-6. For comparison, estimates were also reported using unpartitioned methods. While the unpartitioned method used information on total costs only from observations with complete information (uncensored), the partitioned methods used detailed cost history of the censored and uncensored observations. Both methods are nonparametric and do not adjust for the impact of covariates. Partitioned methods are the preferred approaches because the estimates are robust and precise. ${ }^{12,13}$

Similar to estimating average total costs, censoring poses a challenge for estimating survival times because the entire survival distribution is not observed. The restricted mean survival time (RMST) was estimated for a 5-year follow-up period, which can be written as the following: $\mu(\bar{t})=E[\min \{T, \bar{t}\}]=\int_{0}^{t} S(t)$ $d t$, where $\mu(\bar{t})$ is the RMST, $T$ is time to death, $\bar{t}$ is the follow-up period, and $S(t)$ is the KM survivor curve. ${ }^{14}$ In this case, $\bar{t}=60$ months. Imposing a time limit on survival and cost also avoids issues of identifiability in the marginal distribution of lifetime medical costs. ${ }^{15}$

\section{Selection Bias}

Selection bias is one of the major problems that arises when using a nonrandomized observational dataset. The problem of selection bias on observable factors for average total cost estimates was addressed by using the IPTW method. The inverse of predicted probabilities (propensity scores) of receiving second-line treatment and not receiving second-line treatment were used to adjust for the respective contribution of treated and untreated patients. ${ }^{16-19}$ The propensity scores were estimated using a probit model on the probability of receiving second-line treatment. Covariates included age at the time of diagnosis, marital status, sex, race, place of residence, tumor size, tumor grade, composite index of baseline performance measures, and year of diagnosis. Since the IPTW could be extremely high when probabilities are closer to zero, a normalized IPTW was used (Figure 2).

\section{Cost-Effectiveness Analysis: Incremental Costs, Incremental Survivals, and Incremental Cost-Effectiveness Ratios}

The average incremental costs associated with the receipt of second-line treatment, denoted by $\Delta_{c}$, is the difference between the average costs of patients who received second-line treatment $\left(\mu_{1 c}\right)$ and the average costs of patients who did not receive second-line treatment $\left(\mu_{0 c}\right)$, that is, $\Delta_{c}=\mu_{1 c}-\mu_{0 c}$. Similarly, the incremental survival associated with the receipt of second-line treatment, denoted by $\Delta_{s}$, is the difference between the average months survived by patients who received second-line treatment $\left(\mu_{1 s}\right)$ and the average months survived by patients who did not receive second-line treatment $\left(\mu_{0 s}\right)$, that is, $\Delta_{s}=\mu_{1 s}-\mu_{0 s}$. Incremental costs and incremental survivals were calculated stratified by the type of regimen received in first-line treatment.

The overall cost-effectiveness of second-line treatment can be represented by the incremental cost-effectiveness ratio (ICER), which is a measure of additional cost per unit of health gained. ICER is the ratio of the incremental cost to the incremental survival $\left(\mathrm{ICER}=\Delta_{c} / \Delta_{s}\right)$. ICERs for second-line 


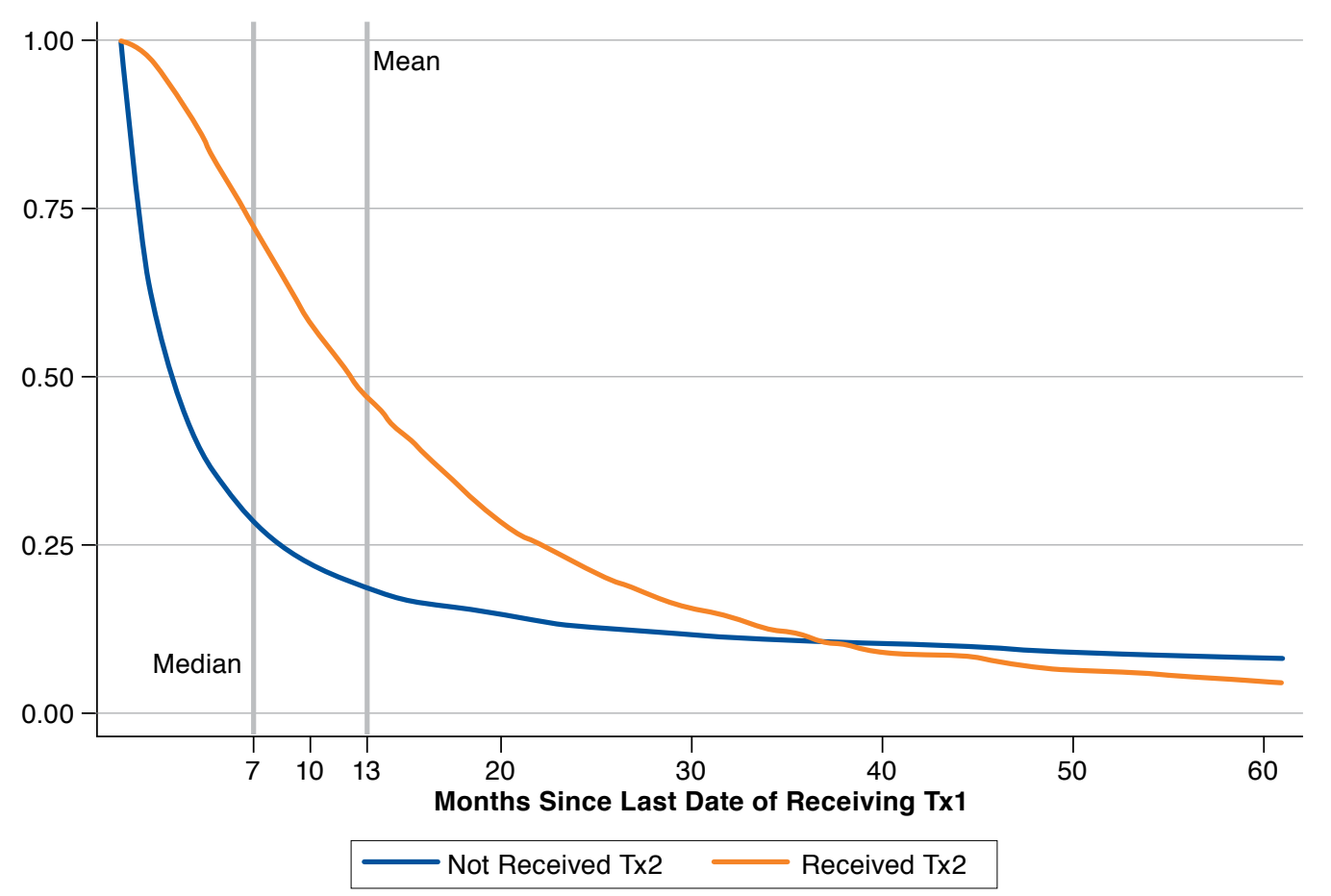

$T \times 1=$ first-line treatment; $T \times 2=$ second-line treatment

treatment were estimated stratified by the type of regimen received in first-line treatment. In order to address skewedness in cost distributions, a robust bootstrap method was used with replacement, which does not rely on distributional assumptions. ${ }^{20,21}$ The bootstrapped values were used to then plot cost-effectiveness acceptability curves (CEACs) for a range of willingness-to-pay (WTP) thresholds given the observed data. The CEAC is a graphical tool that summarizes uncertainties surrounding the estimated ICERs with Bayesian spirit of parameter uncertainty. It shows the likelihood that second-line treatment was cost-effective compared with the alternatives for a range of WTP thresholds.

\section{Results}

Table 2 presents the KM estimates of RMST and incremental survivals by treatment status. It also presents the estimated average total costs and incremental costs associated with the receipt of second-line treatment for the unadjusted estimate (ignoring selection bias) and results after adjusting for selection using the IPTW method.

\section{Survival Benefits}

The estimated mean and median survivals for the entire sample were 13 and 7 months after the last date of receiving first-line treatment. The 2-year, 3-year, and 5-year survival probabilities for patients who received second-line treatments were 24.3\%, $12.2 \%$, and $5 \%$, respectively. For patients who did not receive second-line treatments, the 2-year, 3-year, and 5-year survival probabilities were $13.6 \%, 10.9 \%$, and $8.1 \%$, respectively. The KM survival estimates for patients who received second-line treatments and patients who did not receive second-line treatments are shown in Figure 3.

Patients who received second-line treatment survived for an average of 16.9 months (95\% confidence interval $[\mathrm{CI}]=16.3$ 17.6), whereas those who did not receive second-line treatments survived for an average of 10.2 months ( $95 \%$ CI $=9.5-11$ ). The average incremental survival associated with the receipt of second-line treatment was 6.7 months (95\% CI=5.7-11). However, the gains in survival associated with receipt of second-line treatment vary depending on the type of regimen received in first-line treatment. Patients who started off treatment lines by receiving IRI in first-line treatments gained an average incremental survival of 8.7 months $(95 \% \mathrm{CI}=6.5-11)$ when they received their second-line treatments. The average incremental survival gained from receipt of second-line treatments for patients who received complex regimens such as IROX or targeted therapies in first-line treatment was only 3.9 months (95\% CI =0.0-7.3). 
TABLE 3 Estimated Survivals, Costs, and ICER Per LYG of Second-Line Treatment: Stratified by Type of Regimen Administered in First-Line Treatment

\begin{tabular}{|c|c|c|c|c|c|c|c|c|c|c|}
\hline & & \multirow[b]{2}{*}{ Method } & \multicolumn{2}{|c|}{$\begin{array}{l}\text { First-Line Tx }=5 F U / L V \\
(n=1,392)\end{array}$} & \multicolumn{2}{|c|}{$\begin{array}{l}\text { First-Line Tx = IRI } \\
\qquad(n=651)\end{array}$} & \multicolumn{2}{|c|}{$\begin{array}{c}\text { First-Line Tx }=\mathrm{OX} \\
(\mathbf{n}=2,339)\end{array}$} & \multicolumn{2}{|c|}{$\begin{array}{l}\text { First-Line Tx= Others } \\
(\mathrm{n}=161)\end{array}$} \\
\hline & & & Mean & $95 \% \mathrm{CI}$ & Mean & $95 \%$ CI & Mean & $95 \%$ CI & Mean & 95\% CI \\
\hline \multirow{3}{*}{$\begin{array}{l}\text { Survival } \\
\text { (months) }\end{array}$} & $\begin{array}{l}\text { Received } \\
\text { second-line Tx }\end{array}$ & $\begin{array}{l}\text { Kaplan- } \\
\text { Meier }\end{array}$ & 15.1 & $13.8-16.3$ & 16.2 & $14.4-18.1$ & 18.2 & $17.2-19.3$ & 10.0 & $7.9-12.3$ \\
\hline & $\begin{array}{l}\text { No second-line } \\
\text { Tx }\end{array}$ & $\begin{array}{l}\text { Kaplan- } \\
\text { Meier }\end{array}$ & 8.6 & $7.6-9.6$ & 7.4 & $5.9-9.1$ & 13.1 & $11.8-14.5$ & 6.2 & $3.7-9.3$ \\
\hline & $\begin{array}{l}\text { Incremental } \\
\text { survival }\end{array}$ & $\begin{array}{l}\text { Kaplan- } \\
\text { Meier }\end{array}$ & 6.5 & $4.8-8.1$ & 8.7 & $6.5-11.0$ & 5.1 & $3.5-6.8$ & 3.9 & $0.0-7.3$ \\
\hline \multirow{12}{*}{ Cost, \$ } & \multirow{3}{*}{$\begin{array}{l}\text { Received } \\
\text { Second-line } \\
\text { Tx }\end{array}$} & $\begin{array}{l}\text { Simple } \\
\text { average }\end{array}$ & 96,226 & $88,397-103,015$ & 98,766 & $89,882-107,832$ & 77,470 & $71,227-82,668$ & 146,170 & $117,001-174,714$ \\
\hline & & $\mathrm{B} \& \mathrm{Ta}^{\mathrm{a}}$ & 102,073 & $94,081-109,364$ & 110,241 & $99,344-119,981$ & 91,134 & $84,349-96,957$ & 146,584 & $117,302-175,219$ \\
\hline & & $\operatorname{Lin}^{\mathrm{b}}$ & 98,216 & $90,260-105,424$ & 104,315 & $93,729-113,671$ & 88,462 & $81,685-94,276$ & 130,411 & $103,239-156,705$ \\
\hline & \multirow[t]{3}{*}{$\begin{array}{l}\text { No second-line } \\
\text { Tx }\end{array}$} & $\begin{array}{l}\text { Simple } \\
\text { average }\end{array}$ & 39,821 & $37,647-42,146$ & 36,065 & $33,370-38,951$ & 30,020 & $28,626-31,328$ & 63,753 & $53,881-73,669$ \\
\hline & & $\mathrm{B} \& \mathrm{~T}^{\mathrm{a}}$ & 44,016 & $41,562-46,606$ & 40,573 & $37,487-43,698$ & 37,127 & $35,270-38,899$ & 71,980 & $60,541-83,243$ \\
\hline & & $\operatorname{Lin}^{\mathrm{b}}$ & 39,302 & $36,973-41,649$ & 34,300 & $31,261-37,220$ & 33,094 & $31,281-34,815$ & 59,201 & $49,020-68,949$ \\
\hline & \multirow[t]{3}{*}{$\begin{array}{l}\text { Incremental } \\
\text { cost }\end{array}$} & $\begin{array}{l}\text { Simple } \\
\text { average }\end{array}$ & 56,405 & $48,090-64,030$ & 62,701 & $53,509-72,621$ & 47,450 & $40,927-52,684$ & 82,416 & $51,644-112,183$ \\
\hline & & $\mathrm{B} \& \mathrm{~T}^{\mathrm{a}}$ & 58,057 & $49,109-66,058$ & 69,668 & $58,175-80,804$ & 54,007 & $46,845-59,892$ & 74,604 & $43,622-105,644$ \\
\hline & & $\operatorname{Lin}^{\mathrm{b}}$ & 58,914 & $50,245-66,831$ & 70,015 & $58,916-80,497$ & 55,368 & $48,294-61,290$ & 71,211 & $43,168-99,667$ \\
\hline & \multirow[t]{3}{*}{ ICER/LYG } & $\begin{array}{l}\text { Simple } \\
\text { average }\end{array}$ & 105,983 & $84,634-130,006$ & 87,298 & $70,628-108,605$ & 112,126 & $84,367-150,798$ & 289,984 & $134,810-1,029,969$ \\
\hline & & $\mathrm{B} \& \mathrm{~T}^{\mathrm{a}}$ & 109,026 & $87,903-132,764$ & 96,912 & $79,132-118,496$ & 127,493 & $98,510-168,574$ & 261,485 & $111,739-867,086$ \\
\hline & & Lin $^{\mathrm{b}}$ & 110,621 & $89,560-133,961$ & 97,368 & $80,415-117,965$ & 130,689 & $101,459-171,918$ & 247,951 & $112,629-808,976$ \\
\hline
\end{tabular}

Note: Mean and incremental estimates were based on a bootstrap with 1,000 replications. 95\% CIs were calculated using a percentile method from the same bootstrap.

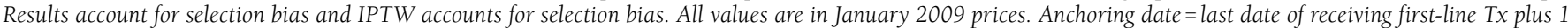
day. Follow-up period $=5$ years (60 months).

a Nonparametric method proposed by Bang and Tsiatis. ${ }^{10}$

${ }^{b}$ Nonparametric method proposed by Lin et al. ${ }^{11}$

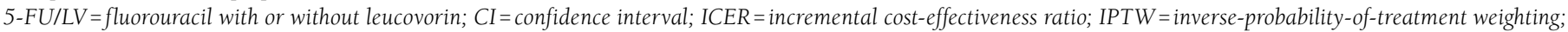
$I R I=$ irinotecan; $L Y G=$ life-year gain; $O X=$ oxaliplatin; $T x=$ treatment.

\section{Estimated Average Total Costs}

The estimated average costs associated with receipt of secondline treatment are shown in Table 2. For the sake of brevity, results are reported from partitioned methods that used detailed cost histories: simple average (naive), B\&T method, and Lin's method. The estimated average total costs varied depending on the methods used to account for censoring and to address selection bias.

While censoring underestimated the average total cost for the treated group and the untreated group, selection bias overestimated the average total cost for the treated group and slightly underestimated the average total cost for the untreated group. When costs were not adjusted for using IPTW, the estimated average 5-year total cost for patients who received second-line treatment was $\$ 103,711, \$ 117,686$, and $\$ 114,076$ using simple average (naive), B\&T method, and Lin's method, respectively. When selection bias using IPTW was accounted for, the estimated average 5-year total cost for second-line treatment recipients was $\$ 89,322, \$ 97,470$, and $\$ 100,530$, respectively, when simple average, Lin's method, and B\&T method were used to adjust for censoring.
For patients who did not receive second-line treatment, the 5 -year average total cost was $\$ 35,068, \$ 36,588$, and $\$ 41,308$ when simple average (naive), Lin's method, and B\&T method, respectively, were used to adjust for censoring. After selection bias on observable factors was addressed, the estimated average total cost was $\$ 35,877, \$ 37,240$, and $\$ 41,963$ when simple average (naive), Lin's method, and B\&T method, respectively, were used to adjust for censoring. The incremental cost associated with receiving a second-line treatment after adjusting selection bias was $\$ 58,567$ and $\$ 60,231$, using $B \& T$ method and Lin's method, respectively. Without adjustment for selection bias, the estimated mean ICERs per life-year gained (LYG) were $\$ 137,384$ and $\$ 139,378$, when censoring was addressed using B\&T method and Lin's method, respectively. When selection bias was controlled for using IPTW, the estimated mean ICERs per LYG became $\$ 105,406$ and $\$ 108,394$, using B\&T method and Lin's method, respectively, for addressing censoring. 
FIGURE 4 Scatter Plots of Incremental Costs and Effects of Second-Line Treatment Stratified by Type of Regimen Administered in First-Line Treatment
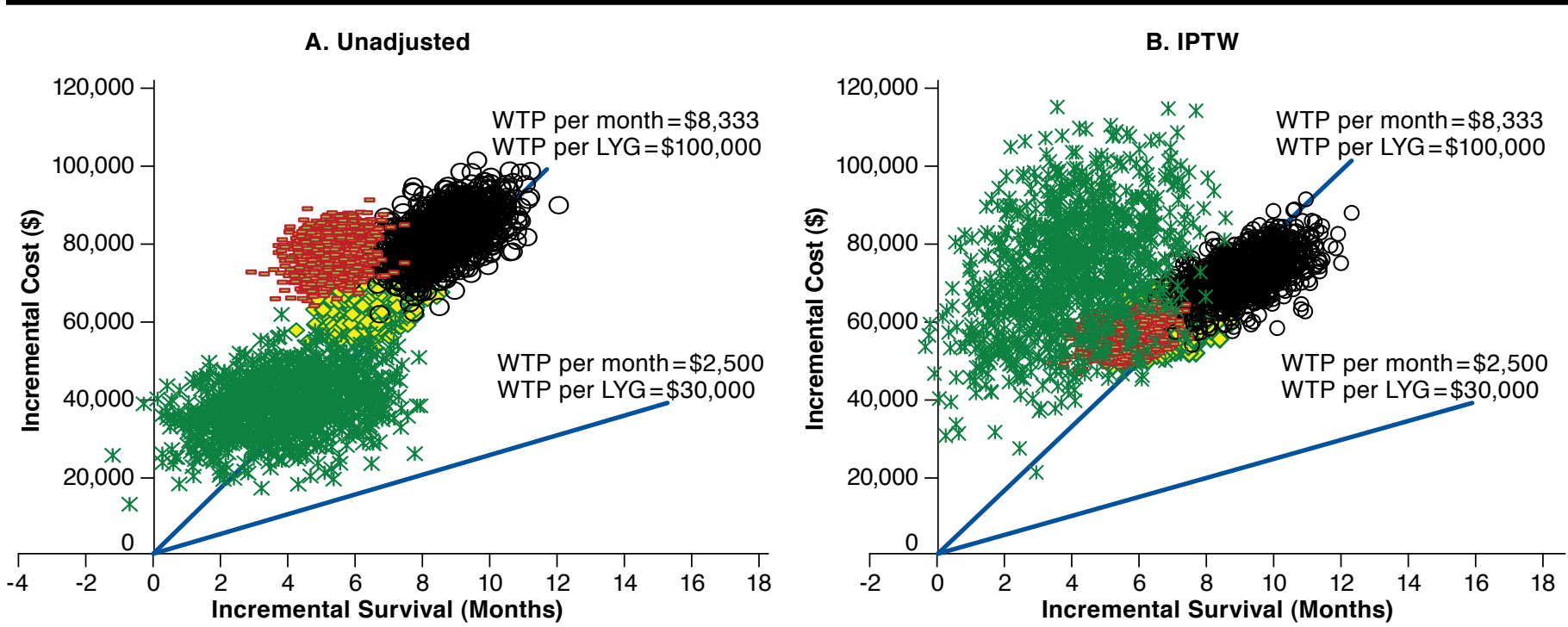

$$
\begin{aligned}
& \diamond \mathrm{Tx} 1=5 \mathrm{FU} / \mathrm{LV} \\
& \circ \mathrm{Tx} 1=\mathrm{IRI} \\
& -\mathrm{Tx} 1=\mathrm{OX} \\
& * \mathrm{Tx} 1=\text { Other }\{\mathrm{IROX}, \mathrm{BIO}\}
\end{aligned}
$$

$$
\begin{aligned}
\diamond \mathrm{Tx} 1 & =5 \mathrm{FU} / \mathrm{LV} \\
\mathrm{T} x 1 & =\mathrm{IRI} \\
\mathrm{T} x 1 & =\mathrm{OX} \\
* \mathrm{~T} 1 & =\text { Other }\{\mathrm{IROX}, \mathrm{BIO}\}
\end{aligned}
$$

Note: Scatter points are based on 1,000 boostrap replications for each strata. Results in (a) baseline do not account for selection bias whereas results in (b) accounts for selection bias. In both cases, censoring is dealt with using Lin et al.'s portioned method.11 All values are in January 2009 prices. Anchoring date $=$ last date of receiving first-line treatment plus 1 day. Follow-up period $=5$ years (60 months).

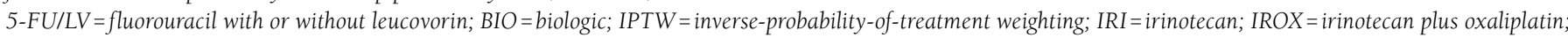
$L Y G=$ life-year gain; $O X=$ oxaliplatin; $T X 1=$ first-line treatment; $W T P=$ willingness to pay.

Table 3 presents the estimated survivals, incremental survivals, average total costs, incremental costs, and ICERs associated with receipt of second-line treatment stratified by the type of regimen administered in first-line treatment. For the sake of brevity, only results obtained after adjusting for selection bias in average total cost using IPTW are presented. For patients who received OX in first-line treatment, the incremental cost associated with receipt of a second-line treatment ranged between $\$ 47,450$ and $\$ 55,368$, and for patients who received IROX or biologic agents alone in first-line treatment, the incremental cost ranges between $\$ 71,211$ and $\$ 82,416$, depending on the method used to address censoring.

With the exception of patients who received other combinations in first-line treatment, the estimated mean ICERs associated with second-line treatment ranged between \$96,912 and $\$ 130,689$ per LYG. The conservative estimate (i.e., the higher average total costs estimate) showed that patients who received IRI, 5FU/LV, and OX in first-line treatment incurred costs of about $\$ 97,368, \$ 110,621$, and $\$ 130,689$, respectively, for a LYG from receiving second-line treatment. However, patients who received other chemotherapy regimens and biologics in first-line treatment on average incurred costs between $\$ 71,211$ and $\$ 74,604$ for only a 4 -month gain in survival from receiving second-line treatment, which implies a mean ICER of above $\$ 250,000$ for an additional LYG.

\section{Discussion}

This study compared costs and survival outcomes of patients who received second-line treatment with patients who did not receive second-line treatment. Incremental survivals, incremental costs, and ICERs associated with second-line treatment were estimated stratified by the type of regimen administered in first-line treatment. Because costs were obtained from Medicare claims, this analysis is from the payer's perspective.

This study showed that receiving second-line treatment significantly increased survival outcome by 6.7 months. However, survival benefits of second-line treatment varied by the type of regimen administered in the first line of treatment. Similarly, costs associated with second-line treatment varied by the type of regimen administered in first-line treatment. For instance, patients who were given OX as first-line treatment incurred lower average total costs in second-line treatment, whereas 


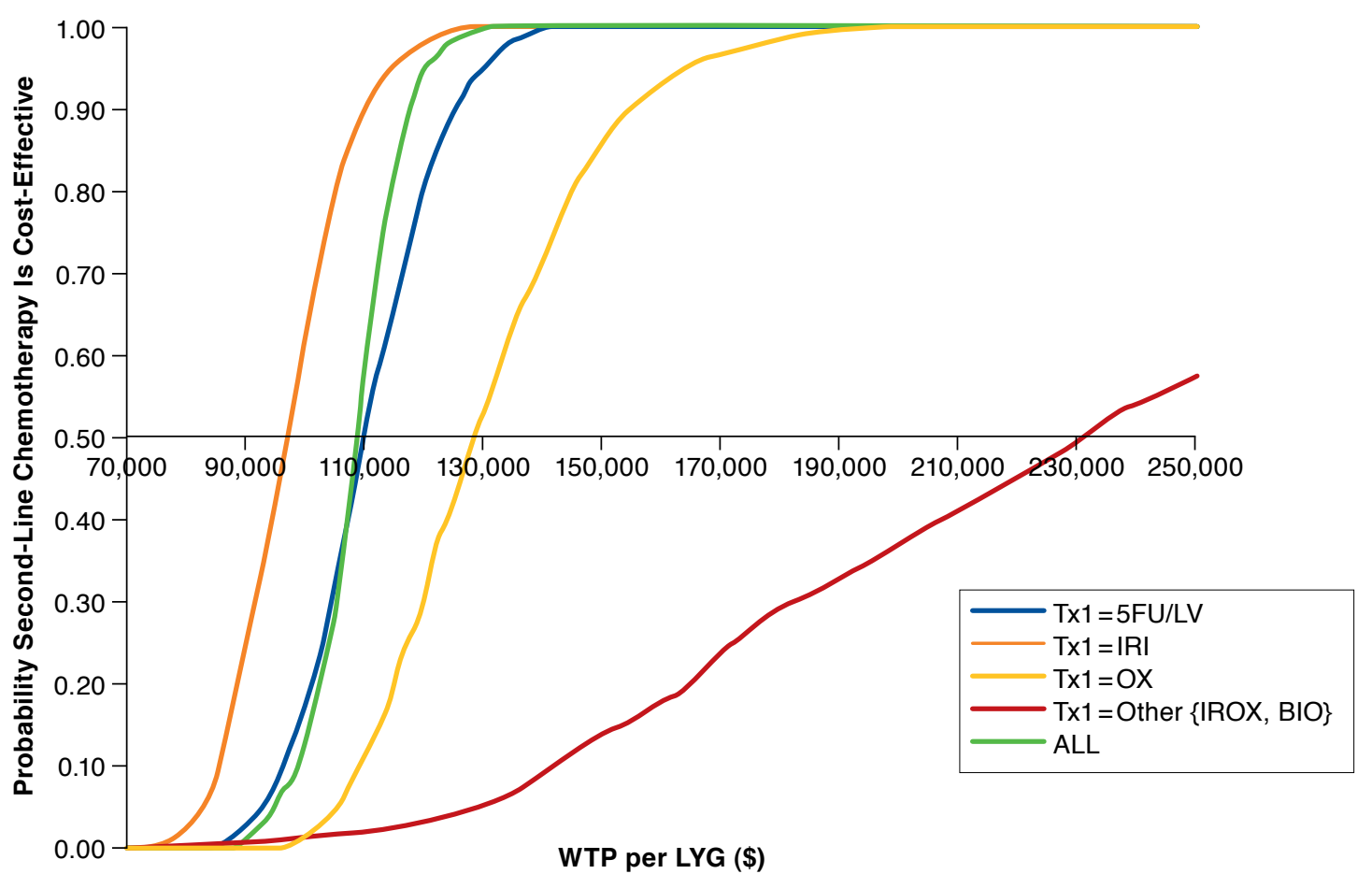

Note: CEACs were based on 1,000 boostrap replications for each strata. Results are from specification that account for selection bias using IPTW and censoring using Lin et al.'s partitioned method. ${ }^{11}$ All values are in January 2009 prices. Anchoring date = last date of receiving first-line Tx plus 1 day. Follow-up period=5 years (60 months). 5-FU/LV = fluorouracil with or without leucovorin; BIO = biologic; $C E A C=$ cost-effectiveness acceptability curve; IPTW=inverse-probability-of-treatment weighting; $I R I=$ irinotecan; $I R O X=$ irinotecan plus oxaliplatin; $L Y G=$ life-year gain; $O X=$ oxaliplatin; $T x 1=$ first-line treatment; $W T P=$ willingness to pay.

patients who were given IROX or a biologic agent alone as firstline treatment incurred higher average total cost in second-line treatment. Furthermore, the results showed variation in the ICER for second-line treatment, depending on the type of regimen administered in first-line treatment. For instance, for all but patients who received other (IROX or biologics) regimens in first-line treatment, bootstrap values of ICERs associated with receipt of second-line treatment were scattered in the northeast region of the cost-effectiveness plane. This result is shown in Figure 4A for the baseline scenario without adjusting for selection bias and in Figure 4B after adjusting for selection bias using IPTW. This result underscores the finding that receiving second-line treatment increases survival outcomes at additional cost, which vary by the type of therapeutic agent received in the first-line of treatment. The scatter plots for 5FU/ LV, IRI, and OX are tighter compared with other combinations. The same pattern is also reflected in the CIs, perhaps because of the small sample size of patients who received other regimens in first-line treatment.

Finally, the assessment of uncertainty associated with estimated mean ICERs using CEAC (Figure 5) shows that, for second-line treatment to be cost-effective $50 \%$ of the time, the required WTP per LYG should be about $\$ 108,000$ for all patients who received second-line treatment. However, if patients received IRI, 5FU/LV, OX, and other regimens in their previous lines of treatment, the WTP for an additional LYG should be $\$ 97,000, \$ 110,000, \$ 128,000$, and $\$ 230,000$, respectively. The CEACs also indicate that there is substantial heterogeneity in the cost-effectiveness and uncertainty surrounding the effectiveness of second-line treatment. Among other factors, it is important to note that the level of uncertainty surrounding estimated incremental costs and ICERs vary considerably depending on statistical method used to address bias and censoring (Figure 6).

\section{Limitations}

Some limitations to this study need to be considered. First, the SEER-Medicare dataset is nonrandomized. Problems associated with a typical observational dataset such as selection bias and censoring in measurement of costs are critical issues that could undermine the validity of estimated parameters. However, SEER-Medicare data are commonly used to conduct economic 


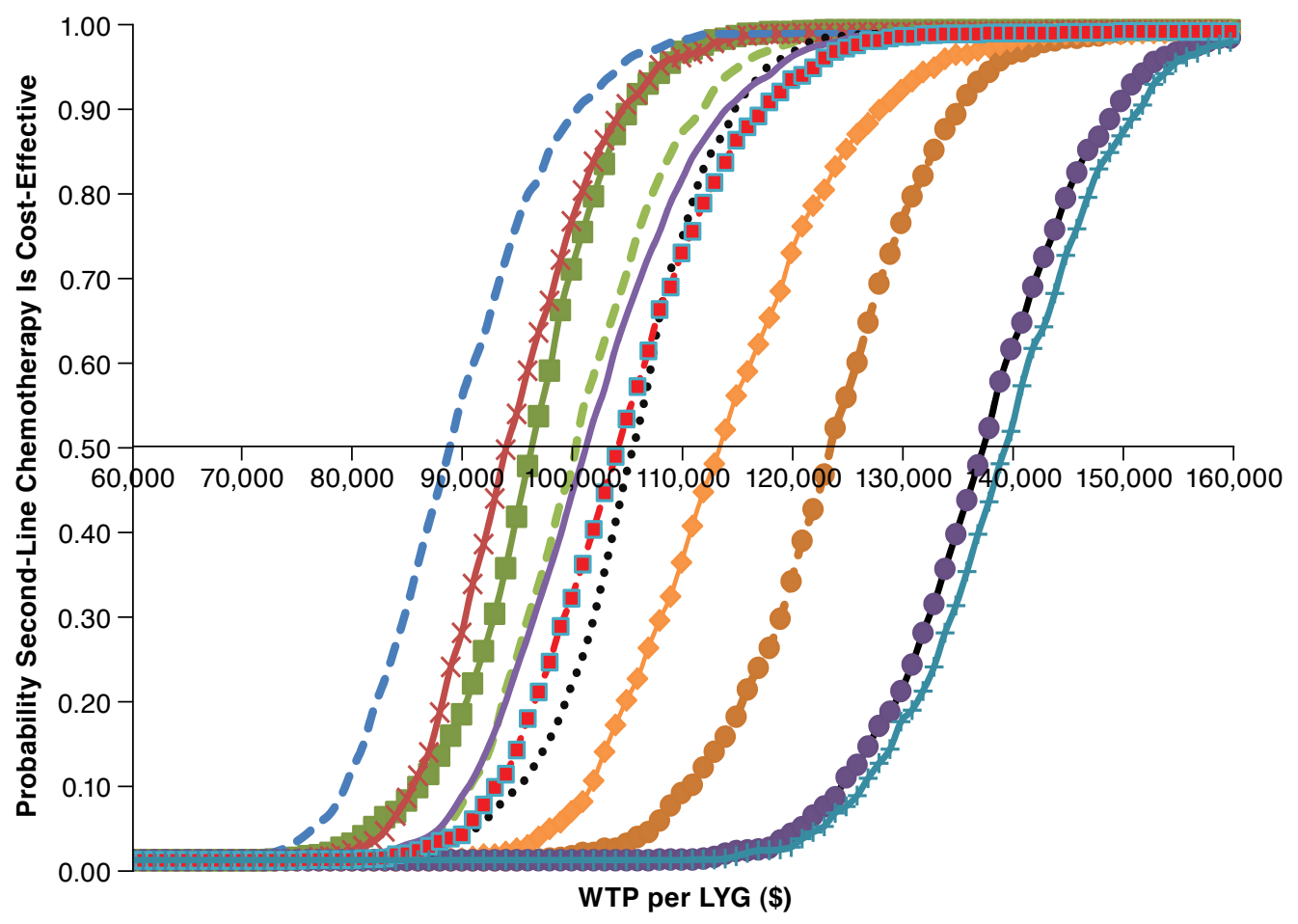

Simple (Unpartitioned)

- Simple (Partitioned)

- Simple (Partitiioned-IPW)

* Simple (Unpartitioned-IPW)

- B\&T (Unpartitioned)a

$\multimap$ B\&T (Unpartitioned-IPW)

- B\&T (Partitioned) $)^{\mathrm{a}}$

...... B\&T (Partitioned-IPW) ${ }^{a}$

Lin (Unpartitioned-IPW)

- - le- Lin (Unpartitioned) ${ }^{\mathrm{b}}$

+ Lin $\left(\right.$ Partitioned) ${ }^{\mathrm{b}}$

a Nonparametric method proposed by Bang and Tsiatis. ${ }^{10}$

${ }^{b}$ Nonparametric method proposed by Lin et al. ${ }^{11}$

$I P W=$ inverse probability weighting; $L Y G=$ life-year gain; $W T P=$ willingness to pay

evaluations and national cost projections. ${ }^{22-24}$ Unlike most RCT studies, the SEER-Medicare database has detailed clinical and claims information on millions of patients, making it one of the most comprehensive datasets available to conduct populationlevel comparative effectiveness studies for the elderly population in the United States.

Second, this analysis does not account for selection on an unobservable dimension. The estimated incremental costs, incremental benefits, and ICERs associated with second-line treatment could be sensitive to unmeasured confounders at patient, provider, and geographic levels. Third, the results are not generalizable to the population outside this sample of elderly patients in the Medicare population diagnosed with metastatic colon cancer between 2003 and 2009. Fourth, the cost estimation did not account for generic versus brand price variations. Such variations could impact the cost-effectiveness analysis, since generic drugs are cheaper than brand names. ${ }^{25}$ Although the drugs considered in this study were brand name during the study period, OX briefly went generic in 2009 until it was reverted back to brand. Also, providers could combine generic with brand-name drugs. Such unaccounted price varia- tions could underestimate incremental costs and ICERs associated with OX, as well as impact the degree of uncertainty.

Last, immortal time bias (survivorship bias) serves as a problem for this study. Patients who survived long enough after first-line treatment were more likely to receive secondline treatment (survivors). Patients whose conditions were deteriorating could discontinue intensive therapy after first-line treatment in favor of supportive/palliative care. As a result, the estimated second-line survival benefits could be overstated.

\section{Conclusions}

Among elderly metastatic colon cancer patients, costs and survival benefits associated with the receipt of second-line treatment depend on the type of regimen administered in the firstline treatment. The estimated incremental survivals, incremental costs, and ICERs vary by the type of regimen administered in the first-line treatment. Patients who received second-line treatment lived for an additional 6.7 months compared with patients who did not receive second-line treatment. However, the gain in survival varies between 4 and 9 months, depending on the type of regimen received in the first-line treatment. 
The incremental cost associated with the receipt of secondline treatment was $\$ 60,231$ but ranged between $\$ 47,450$ and $\$ 82,416$, depending on the type of regimen administered in the first-line treatment. Combining unadjusted survival benefits and costs, the ICER per LYG associated with receipt of second-line treatment ranged between $\$ 96,912$ and $\$ 247,951$, depending on the type of regimen administered in the firstline treatment. This study's results were robust to different statistical models. However, unmeasured confounders were not accounted for, and interpretation of results should be limited to the SEER-Medicare population. The ultimate conclusion, however, is that when therapies are administered in a sequential manner, the cost-effectiveness of the second line of therapy depends on what was administered in the first line of therapy.

\section{Authors}

ANDINET WOLDEMICHAEL, PhD, Department of Pharmaceutical Health Services, University of Maryland School of Pharmacy, Baltimore. EBERECHUKWU ONUKWUGHA, MSc, $P h D$, and C. DANIEL MULLINS, PhD, Pharmaceutical Health Services Research, University of Maryland School of Pharmacy, Baltimore. BRIAN SEAL, RPh, MBA, PhD, Bayer Healthcare Pharmaceuticals, Wayne, New Jersey, and NADER HANNA, MD, Department of Surgery, Division of General and Oncologic Surgery, University of Maryland School of Medicine, Baltimore.

AUTHOR CORRESPONDENCE: Andinet Woldemichael, PhD, Department of Pharmaceutical Health Services, University of Maryland School of Pharmacy, 220 Arch St., Baltimore, MD 21201. Tel.: 410.706.6627; Fax: 410.706.5394;

E-mail: andinet.michael@gmail.com.
The authors acknowledge the efforts of the Applied Research Program, National Cancer Institute; the Office of Research, Development, and Information, Centers for Medicare \& Medicaid Services; Information Management Services; and the Surveillance, Epidemiology, and End Results Program tumor registries in the creation of the SEER-Medicare database.

The authors acknowledge Kaloyan Bikov and Corinne Woods, who helped with constructing the analytical data file, and Laura Bogart, who proofread the manuscript. The Pharmaceutical Research Computing Center, housed in the Pharmaceutical Health Services Research Department at the University of Maryland School of Pharmacy, was instrumental in the construction of the analytical data used in this analysis.

\section{REFERENCES}

1. Schrag D. The price tag on progress-chemotherapy for colorectal cancer. N Engl J Med. 2004;351(4):317-19.

2. Hurwitz H, Fehrenbacher L, Novotny W, et al. Bevacizumab plus irinotecan, fluorouracil, and leucovorin for metastatic colorectal cancer. N Engl J Med. 2004;350(23):2335-42.

3. Zheng Z, Hanna N, Onukwugha E, Reese E, Seal B, Mullins CD. Does the type of first-line regimens influence the receipt of second-line chemotherapy treatment? An analysis of 3211 metastatic colon cancer patients. Cancer Med. 2014;3(1):124-33.

4. Iveson TJ, Hickish T, Schmitt C, Van Cutsem E. Irinotecan in second-line treatment of metastatic colorectal cancer: improved survival and cost-effect compared with infusional 5-FU. Eur J Cancer. 1999;35(13):1796-804.

5. Levy-Piedbois C, Durand-Zaleski I, Juhel H, Schmitt C, Bellanger A, Piedbois P. Cost-effectiveness of second-line treatment with irinotecan or infusional 5-fluorouracil in metastatic colorectal cancer. Ann Oncol. 2000;11(2):157-61.

6. Tappenden P, Jones R, Paisley S, Carroll C. The cost-effectiveness of bevacizumab in the first-line treatment of metastatic colorectal cancer in England and Wales. Eur J Cancer. 2007;43(17):2487-94.

7. Starling N, Tilden D, White J, Cunningham D. Cost-effectiveness analysis of cetuximab/irinotecan vs active/best supportive care for the treatment of metastatic colorectal cancer patients who have failed previous chemotherapy treatment. Br J Cancer. 2007;96(2):206-12.

8. National Cancer Institute (NCI). Colon cancer treatment-patient version (PDQ). Updated July 22, 2015. Available at: http://cancer.gov/cancertopics/ pdq/treatment/colon/Patient. Accessed March 14, 2016.

9. Bikov KA, Mullins CD, Seal B, Onukwugha E, Hanna N. Algorithm for identifying chemotherapy/biological regimens for metastatic colon cancer in SEER-Medicare. Med Care. 2013;53(8):e58-e64.

10. Bang H, Tsiatis A. Estimating medical costs with censored data. Biometrika. 2000;87(2):329-43.

11. Lin DY, Feuer EJ, Etzioni R, Wax Y. Estimating medical costs from incomplete follow-up data. Biometrics. 1997;53(2):419-34.

12. Raikou M, McGuire A. Estimating medical care costs under conditions of censoring. J Health Econ. 2004;23(3):443-70.

13. Wijeysundera HC, Wang X, Tomlinson G, Ko DT, Krahn MD.

Techniques for estimating health care costs with censored data: an overview for the health services researcher. Clinicoecon Outcomes Res. 2012:4:145-55.

14. Royston P, Parmar MK. Restricted mean survival time: an alternative to the hazard ratio for the design and analysis of randomized trials with a time-to-event outcome. BMC Med Res Methodol. 2013;13(1):152.

15. Huang Y. Cost analysis with censored data. Med Care. 2009;47(7 Suppl 1): S115-19.

16. Hernán MA, Robins JM. Estimating causal effects from epidemiological data. J Epidemiol Community Health. 2006;60(7):578-86.

17. Wooldridge JM. Inverse probability weighted estimation for general missing data problems. J Econometrics. 2007;141(2):1281-301. 
18. Curtis LH, Hammill BG, Eisenstein EL, Kramer JM, Anstrom KJ. Using inverse probability-weighted estimators in comparative effectiveness analyses with observational databases. Med Care. 2007;45(10 Suppl 2):S103-07.

19. Imbens $G$, Wooldridge J. Recent developments in the econometrics of program evaluation. J Econ Lit. 2009;47(1):5-86.

20. Wakker P, Klaassen MP. Confidence intervals for cost/effectiveness ratios. Health Econ. 1995;4(5):373-81.

21. Indurkhya A, Gardiner JC, Luo Z. The effect of outliers on confidence interval procedures for cost-effectiveness ratios. Stat Med. 2001;20(9-10): 1469-77.
22. Yabroff KR, Borowski L, Lipscomb J. Economic studies in colorectal cancer: challenges in measuring and comparing costs. J Natl Cancer Inst Monogr. 2013;2013(46):62-78

23. Mariotto AB, Yabroff KR, Shao Y, Feuer EJ, Brown ML. Projections of the cost of cancer care in the United States: 2010-2020. J Natl Cancer. 2011;103(2):117-28

24. Howlader N, Noone AM, Krapcho M, et al., eds. SEER cancer statistics review, 1975-2010. National Cancer Institute. Bethesda, MD, based on November 2012 SEER data submission, posted to the SEER website, April 2013. Available at: http://seer.cancer.gov/archive/csr/1975_2010/. Accessed Februay 24, 2016.

25. Shih YT, Han S, Cantor SB. Impact of generic drug entry on cost-effectiveness analysis. Med Decis Making. 2005;25(1):71-80. 